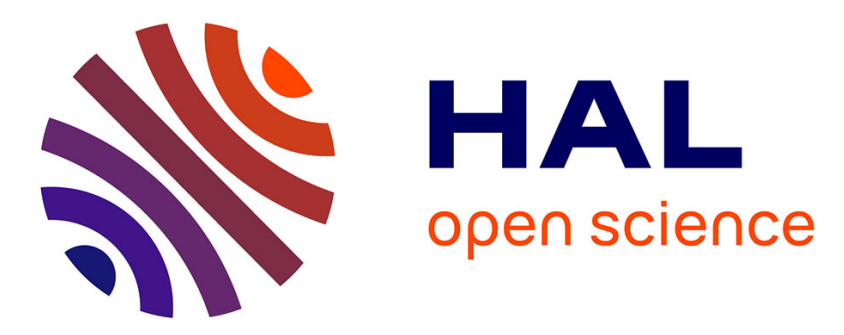

\title{
Audiological Results and Quality of Life of Sophono Alpha 2 Transcutaneous Bone-Anchored Implant Users in Single-Sided Deafness
}

\author{
Daniele Bernardeschi, Francesca Yoshie Russo, Yann Nguyen, Eric Vicault, \\ Jonathan Flament, Deborah Bernou, Olivier Sterkers, Isabelle Mosnier
}

\section{To cite this version:}

Daniele Bernardeschi, Francesca Yoshie Russo, Yann Nguyen, Eric Vicault, Jonathan Flament, et al.. Audiological Results and Quality of Life of Sophono Alpha 2 Transcutaneous Bone-Anchored Implant Users in Single-Sided Deafness. Audiology and Neurotology, 2016, 21 (3), pp.158 - 164. 10.1159/000445344. hal-01400669

\section{HAL Id: hal-01400669 \\ https://hal.sorbonne-universite.fr/hal-01400669}

Submitted on 22 Nov 2016

HAL is a multi-disciplinary open access archive for the deposit and dissemination of scientific research documents, whether they are published or not. The documents may come from teaching and research institutions in France or abroad, or from public or private research centers.
L'archive ouverte pluridisciplinaire HAL, est destinée au dépôt et à la diffusion de documents scientifiques de niveau recherche, publiés ou non, émanant des établissements d'enseignement et de recherche français ou étrangers, des laboratoires publics ou privés. 


\section{AUDIOLOGICAL RESULTS AND QUALITY OF LIFE OF SOPHONO}

\section{ALPHA 2 TRANSCUTANEOUS BONE-ANCHORED IMPLANT USERS}

3 IN SINGLE-SIDED DEAFNESS

4 RUNNING TITLE: SOPHONO ALPHA 2 IN SSD

5 Daniele Bernardeschi ${ }^{1,2,3}$, Francesca Yoshie Russo ${ }^{1,2,3}$, Yann Nguyen ${ }^{1,2,3}$, Eric Vicault ${ }^{4}$,

6 Jonathan Flament ${ }^{1,5}$, Deborah Bernou ${ }^{6}$, Olivier Sterkers ${ }^{1,2,3}$ and Isabelle Mosnier ${ }^{1,2,3}$

7 1. AP-HP, Groupe Hospitalier Pitié-Salpêtrière, Unité Otologie, Implants auditifs et Chirurgie

8 de la base du crâne, 75013, Paris, France

9 2. Sorbonne Universités, UPMC Univ Paris 06, UMR-S 1159, «Réhabilitation chirurgicale

10 mini-invasive et robotisée de l'audition», F-75005, Paris, France

3. INSERM UMR-S 1159, «Réhabilitation chirurgicale mini-invasive et robotisée de l'audition», F-75018 France

4. AP-HP, Lariboisière Hospital, Clinical Research Center, Paris, France

6. Collin ${ }^{\circledR}$ Ltd, Bagneux, France

\section{Corresponding author:}

Daniele Bernardeschi, MD, PhD

Otology, auditory implants and skull base surgery department

Pitié-Salpêtrière Hospital

50/52 Bd Vincent Auriol - 75013 - Paris - France

Tel: $+33(0) 142162603$

Fax: + $33(0) 142162605$ 
$1 \quad$ E-mail : daniele.bernardeschi@psl.aphp.fr

2

3 Disclosure statement: Authors disclose any sponsorship or funding arrangements relating to

4 their research. Deborah Bernou works as biomedical engineer for Collin Ltd, France, a local 5 distributor of Alpha system.

6 


\section{ABSTRACT}

2 Single-sided deafness (SSD) represents one of the most difficult audiological condition to

3 rehabilitate. The aim of this prospective study was to evaluate the audiological benefits and

4 the quality of life when upgrading to Sophono Alpha $2^{\circledR}$ (Boulder, Colo., USA) external

5 processor, patients affected by SSD previously users of Alpha $1^{\circledR}$. Nine patients have been

6 included. They underwent physical examination, free-field speech audiometry at 40dB and 60

$7 \mathrm{~dB}$, hearing in noise test (Hirsch's test and squelch test), Glasgow benefit inventory (GBI)

8 questionnaire, and patient's satisfaction specific questionnaire with Alpha $1^{\circledR}$. Afterwards, the

9 Alpha $2^{\circledR}$ external processor was delivered to all patients and the above mentioned protocol was repeated after 1 month with the Alpha $2^{\circledR}$. A statistically significant improvement was found in the speech discrimination score at $40 \mathrm{~dB}$ and in the squelch test when using the Alpha $2^{\circledR}$ external processor compared to the Alpha $1^{\circledR}$. Concerning clinical tolerance, the specific questionnaire and the GBI, the differences were not significant. The new Alpha $2^{\circledR}$ external processor represents a safe and effective device for the rehabilitation of SSD and there is an audiological benefit from upgrading to Alpha $2^{\circledR}$ external processor patients previously users of the Alpha $1^{\circledR}$. The improvement in the quality of life is similar to other bone-anchored hearing devices.

Keywords: single-sided deafness, transcutaneous bone-anchored hearing device, Hearing aids, bone conduction, implantable hearing aids, hearing loss 


\section{INTRODUCTION}

3 Single sided deafness (SSD) affects the quality of life of the patients, since they experience

4 discomfort in difficult situations such as hearing in noise or localizing the sound [Douglas et

5 al., 2007]. The loss of hearing in one ear suppresses the interaural time and sound level

6 differences that are responsible of localization [Kitterick et al., 2014]. Patient affected by SSD

7 also show the effect of the acoustic shadow of the head [Carlile, 2006] which is the cause of these difficulties [Van Wanrooij, and Van Opstal, 2004]. Finally, listening to sounds coming from impaired side is also challenging for these patients.

Many studies have been carried out in order to define which was the optimal treatment for this pathological condition. Contralateral rerouting of signal (CROS) hearing aids [Finbow et al., 2015; Bosman et al., 2003; Hol et al., 2005; Wazen et al., 2003], and percutaneous boneanchored hearing devices [Faber et al., 2013; Zeitler et al., 2012; Stewart et al., 2011; Martin et al., 2010] have been proposed for the rehabilitation of SSD with different results. More recently, cochlear implant has been suggested for the rehabilitation of SSD [van Zon et al., 2015; Erbele et al., 2015; Arndt et al., 2011; Vermeire, and Van de Heyning, 2009].

The Sophono Alpha ${ }^{\circledR}$ system (Sophono, Boulder, Colo., USA) is a transcutaneous bone conductive hearing device: the major advantage of this system, compared to the percutaneous ones, is the presence of the intact skin that dramatically reduced the skin related complications [Wazen et al., 2008]. The use of Alpha $1^{\circledR}$ system has already been described for conductive or mixed hearing loss, in adults and in children [Sylvester et al., 2013; Escorihuela-García et al., 2014; Siegert, and Kanderske, 2013; Hol et al., 2013; Magliulo et al., 2014; Denoyelle et al., 2013]. The first prospective clinical study comparing the Alpha $1^{\circledR}$ system and CROS hearing aids in SSD has been recently published [Leterme et al., 2015]. Authors showed that the Alpha $1{ }^{\circledR}$ was preferred to CROS in $72 \%$ of patients and that the hearing performance 
1 were significantly improved when using the Alpha $1^{\circledR}$ system compared to unaided condition,

2 whereas there were not statistically significant differences when comparing the audiological

3 outcomes of the 2 devices.

4 Recently, the new Sophono Alpha $2^{\circledR}$ external processor has been released: the main

5 differences between the Alpha 1 and the Alpha 2 are the following:

$6 \quad-\quad$ A more square and little shape (Fig. 1A and 1B)

$7 \quad-\quad$ The presence of 8 digital channels (instead of 4) with 8 programmable settings to facilitate program changing in different environments

- The presence of 2 microphones (instead of 1 omnidirectional); the first one is directional and the second is omnidirectional processor, when upgrading them to the new Alpha $2^{\circledR}$ external processor. 


\section{MATERIALS AND METHODS}

2 This prospective clinical study was authorized by the hospital ethical committee and all

3 patients gave the written consent for the use of their clinical data. Collin ${ }^{\circledR}$ Ltd. (Bagneux,

4 France), local distributor of Alpha system, supported this study by providing the Alpha $2^{\circledR}$

5 external processor to all patients.

6 The Sophono Alpha ${ }^{\circledR}$ system consists in one external processor and one implantable twin

7 magnet (Fig. 1C). The external processor contains a bone conductive oscillator which is mounted on two external magnets. The implantable twin magnets are encapsulated in a titanium case which is fixed to the skull with five little arms.

Nine patients were included in this protocol (Tab. 1). All patients had been using the Alpha $1^{\circledR}$ system for at least 1 year. There were 5 males and 4 females. The mean age was $50 \pm 9.9$ years (mean \pm SD, range 40/65 years); the right side was involved in 5 cases, the left side in 4 eases. The etiology of unilateral hearing loss was vestibular schwannoma surgery (22\%), sudden sensorineural hearing loss $(22 \%)$, chronic otitis (11.2\%) with ipsilateral profound hearing loss, head trauma (11.2\%), ototoxicity (11.2\%), mumps (11.2\%), congenital (11.2\%). The mean air conduction threshold (mean of the frequencies $500,1000,2000$ and $4000 \mathrm{~Hz}$ ) of the contralateral ear was $21 \pm 9 \mathrm{~dB}$ and the bone conduction threshold was $14 \pm 8.6 \mathrm{~dB}$ (Tab. 1). At the first visit, a physical examination of the skin over the implant was performed (normal or hyperemic). The strength of the magnet, ranging from 0 to 7 was noted. The strength of the magnet was the lowest strength that allowed the uncoupling of the external device when a strength ranging from 1 and $1.5 \mathrm{~N}$ was applied with a specific tool. The total hour of wearing the external processor per day was analyzed, as well as the regularity of wearing the external processor (everyday or occasionally in particular situation as in noisy environment, in social activities etc.). 


\section{Audiological tests}

All tests were performed in an audiometric insonorized room. The mean air conduction (AC) and bone conduction $(\mathrm{BC})$ thresholds were calculated using headphone (mean of the frequencies 500,1000, 2000 and $4000 \mathrm{~Hz}$ ). In the free-field test as well as in noise, 3 speakers $\underline{\text { were used placed at } 1 \mathrm{~m} \text { from the patient (in front, left and right ear) }}$

Free-field speech audiometry in quiet using French Lafon's monosyllabic words delivered at $\underline{40 \text { and } 60 \mathrm{~dB} \text { to the deaf side was performed in unaided (UA) condition and without Alpha } 1^{\circledR}}$ device. The percentage of correct responses was noted.

$\underline{\text { In noise, two tests were performed depending of the respective presentation of the noise and }}$ stimulation: The Hirsch's test [Hirsch, and Anderson, 1980] (white $65 \mathrm{~dB}$ noise presented frontally and French Lafon's monosyllabic words of increasing intensity delivered to the deaf side) was performed in UA condition and without Alpha $1^{\circledR}$ and the speech reception $\underline{\text { threshold (SRT) was noted. The squelch test using white noise at } 65 \mathrm{~dB} \text { delivered to the deaf }}$ $\underline{\text { side and monosyllabic words of increasing intensity presented frontally was performed in UA }}$ condition and with Alpha $1^{\circledR}$, recording the SRT. Both tests aimed to measure the efficacy of the system for the transfer of sound information to the contralateral side.

\section{Questionnaires}

Patient satisfaction with Alpha $1^{\circledR}$ was evaluated by Glasgow Benefit Inventory (GBI) [Robinson et al., 1996] and by a specific questionnaire. The GBI was divided in 3 subgroups (general, social support and physical health) and the results were reported from -100 to +100 for each subgroup and for the global score. The specific questionnaire investigated the satisfaction of wearing the device through a Likert scale ranging from 0 to $4(0=$ very dissatisfied; $1=$ somewhat dissatisfied; $2=$ neither satisfied nor dissatisfied; $3=$ satisfied; $4=$ very satisfied). 
1 Afterwards, the Alpha $2^{\circledR}$ external processor was delivered to all patients, and all the test and

2 questionnaires described above were repeated after 30 days with the Alpha $2^{\circledR}$ processor. The

3 same method of fitting was used across the patients and this fitting was normalized with air

4 conduction pure-tone average, as well as the same magnetic strength was maintained between

$5 \quad$ Alpha 1 and Alpha 2.

\section{$6 \quad$ Statistical analysis}

7 Values were expressed as means \pm SD. The results obtained with the two external processors

8 were analyzed by a paired t-test or by non-parametric Wilcoxon test because of the small

9 number of patients. Qualitative data were compared using Mc Nemar test. A p value $<0.05$

10 was considered significant. 


\section{RESULTS}

2 All patients completed the protocol.

3 Population

4 There were 5 males and 4 females. The mean age was $50 \pm 9.9$ years (mean $\pm \mathrm{SD}$, range

$5 \quad 40 / 65$ years); the right side was involved in 5 cases, the left side in 4 cases. The etiology of

6 unilateral hearing loss was vestibular schwannoma surgery (22\%), sudden sensorineural

7 hearing loss (22\%), chronic otitis (11.2\%) with ipsilateral profound hearing loss, head trauma

$8 \quad(11.2 \%)$, ototoxicity (11.2\%), mumps (11.2\%), congenital (11.2\%).

9 At the first visit with the Alpha $1^{\circledR}$, the local skin over the implant was evaluated normal in 8

10

11

12 cases and hyperemic in 1 case. The mean strength of the external magnet was $5 \pm 1.7 \mathrm{~N}$. The mean wearing time per day was $5 \pm 4.5$ hours and the patients had a regular daily use of the Alpha $1^{\circledR}$ in 5 cases, and an occasional use in 4 cases.

With the Alpha $2^{\circledR}$, the local skin was evaluated normal in 7 cases and hyperemic in 2 cases.

The strength of the external magnet was $5 \pm 1.9$, and the wearing time was $7 \pm 4.3$ hours, values that were similar to those obtained with Alpha $1^{\circledR}$. One more patient used the Alpha $2^{\circledR}$ regularly (6 instead of 5 for Alpha $\left.1^{\circledR}\right)$.

\section{Audiological tests}

The mean air conduction threshold (mean of the frequencies 500,1000, 2000 and $4000 \mathrm{~Hz}$ ) of the contralateral ear was $21 \pm 9 \mathrm{~dB}$ and the bone conduction threshold was $14 \pm 8.6 \mathrm{~dB}$ (Tab. $\underline{1)}$.

The mean speech discrimination score (Fig. 2A) using monosyllabic words delivered at $40 \mathrm{~dB}$ $(73 \%)$ and at $60 \mathrm{~dB}(98 \%)$ was similar in UA condition and with Alpha $1^{\circledR}$. Similarly, no difference in SRT in both Hirsch's test $(58 \mathrm{~dB})$ and squelch test $(54 \mathrm{~dB})$ was observed between the UA condition and with the Alpha $1^{\circledR}$ (Fig. 2B). 
1 At the 1 month audiological tests with Alpha $2^{\circledR}$, using monosyllabic words at $40 \mathrm{~dB}$, the

2 speech discrimination score was $82 \pm 19.7 \%$ : the mean gain was $8 \pm 8 \%$ and this difference

3 was significant (paired t-test; $\mathrm{p}<0.005$ ) (Fig. 2A).

4 At $60 \mathrm{~dB}$, the speech discrimination score was $100 \pm 0.7 \%$ with Alpha $2^{\circledR}$, a value not

5 different to that observed with Alpha $1^{\circledR}$.

6 Regarding the Hirsch's test (Fig. 2B), the SRT with Alpha $2^{\circledR}$ was similar to that observed

7 with Alpha $1^{\circledR}$ but it was improved by $4 \pm 4 \mathrm{~dB}$ ( $\mathrm{p}<0.02$, paired t-test) compared to UA condition.

8 For the squelch test, the SRT with Alpha $2^{\circledR}$ improved of $1.4 \pm 1 \mathrm{~dB}(\mathrm{p}<0.02$, paired t-test $)$

9 compared to that measured with Alpha $1^{\circledR}$.

\section{Questionnaires}

The GBI global score with Alpha $1^{\circledR}$ was $11 \pm 12.9$, and the scores for the subscales were $12 \pm$ $15.6,15 \pm 22.7$ and $6 \pm 22.6$ for the general, social support and physical health respectively; Fig. 5). Patient's satisfaction stood at $2 \pm 1.2$ using the specific questionnaire.

Regarding the GBI score, with the use of Alpha $2^{\circledR}$ device we obtained a value of $14 \pm 11.0$ for the global score and $18 \pm 18.3,18 \pm 22.7,-4 \pm 11.1$ for the general, social support and physical health subscales respectively. There was no significance when comparing these data to the results with the Alpha $1 \circledR$ processor (Fig. 3, Wilcoxon test).

The specific questionnaire revealed a score of $3 \pm 0.3$ with the Alpha $2^{\circledR}$ and the difference with the score obtained with the Alpha $1^{\circledR}$ was not significant (Wilcoxon test). 


\section{DISCUSSION}

2 The SSD certainly represents one of the most difficult clinical condition for hearing

3 rehabilitation: Studies analyzing the results of BAHA showed that the hearing outcomes and the patient's satisfaction were significantly poorer in patients affected by SSD than in those affected by conductive of mixed hearing loss [Tringali et al., 2008; Martin et al., 2010]. Furthermore, when using a trans-cutaneous bone conductive hearing device, the presence of an intact skin, although beneficial from a clinical point of view, attenuates sound transmission especially in high frequencies [Verstraeten et al., 2009; Kurz et al., 2014], and this could decrease the efficiency of this device when used in SSD.

Sophono Alpha system is a bone conductive transcutaneous implant that transmits the vibrations of the external processor thought an intact skin, by means of a magnetic coupling. It has been described firstly by Siegert et al. [Siegert, 2011] and since, other papers have been published concerning the use of this device in different pathological condition [Denoyelle et al., 2013; Hol et al., 2013; Sylvester et al., 2013; Magliulo et al., 2014; Escorihuela-García et al., 2014; Leterme et al., 2015; Siegert, and Kanderske, 2013].

The use of Alpha $1^{\circledR}$ system has already been described in conductive or mixed hearing loss in adults and in children [Sylvester et al., 2013; Escorihuela-García et al., 2014; Siegert, and Kanderske, 2013; Hol et al., 2013; Magliulo et al., 2014; Denoyelle et al., 2013]. No studies have been yet published in the English literature with the use of Alpha $2^{\circledR}$. After the release of the new external processor Alpha $2^{\circledR}$, we aimed to study if, regardless the technological improvements of this new external processor, there were audiological improvements with the use of this new device compared to the previous Alpha $1^{\circledR}$. First of all, the new external processor is safe: no increase in adverse skin reactions was observed and the patient's satisfaction improved, even if this was not significant in the specific questionnaire and in the GBI due to the limited number of patient. Nevertheless the 
1 results of the GBI questionnaires are similar to those obtained with the use of other bone

2 anchored hearing devices [Faber et al., 2013; Saroul et al., 2013] in SSD.

3 From an audiological point of view, as expected, the benefits of Alpha $2^{\circledR}$ are significant at 40

$4 \mathrm{~dB}$ stimulation and this could be useful in improving speech perception of the deaf side. At 65

$5 \mathrm{~dB}$ this difference is not significant probably because, at this level of stimulation, the normal

6 hearing ear is stimulated as well. Regarding hearing-in-noise tests, we observed a significant

7 differences for the squelch test: this test reflects the reduced masking of the speech by the

8 noise [Snik et al., 2015] and the improvement is probably due to the presence of the 2

9 microphones in the Alpha $2^{\circledR}$ external processor. For the Hirsch's test, even if there was an improvement in the scores, this was not significant probably because of the low number of cases. Anyway, if compared to unaided condition, the improvement with Alpha $2{ }^{\circledR}$ was significant.

In conclusion, the new external processor Alpha $2^{\circledR}$ improves the hearing performance in patients with SSD compared to the previous Alpha $1^{\circledR}$ external processor; since this pathological condition is one of the most challenging to rehabilitate, the upgrade revealed beneficial for the patients. 


\section{REFERENCES}

2

Arndt S, Aschendorff A, Laszig R, Beck R, Schild C, Kroeger S, et al.: Comparison of pseudobinaural hearing to real binaural hearing rehabilitation after cochlear implantation in patients with unilateral deafness and tinnitus. Otol Neurotol 2011;32:39-47.

Bosman AJ, Hol MKS, Snik AFM, Mylanus EAM, Cremers CWRJ: Bone-anchored hearing aids in unilateral inner ear deafness. Acta Otolaryngol 2003;123:258-260.

Carlile S: Listening to the world around us. 2006;34:5-11.

Denoyelle F, Leboulanger N, Coudert C, Mazzaschi O, Loundon N, Vicaut E, et al.: New closed skin bone-anchored implant: preliminary results in 6 children with ear atresia. Otol Neurotol 2013;34:275-281.

Douglas SA, Yeung P, Daudia A, Gatehouse S, O’Donoghue GM: Spatial hearing disability after acoustic neuroma removal. Laryngoscope 2007;117:1648-1651.

Erbele ID, Bernstein JGW, Schuchman GI, Brungart DS, Rivera A: An initial experience of cochlear implantation for patients with single-sided deafness after prior osseointegrated hearing device. Otol Neurotol 2015;36:e24-29.

Escorihuela-García V, Llópez-Carratalá I, Pitarch-Ribas I, Latorre-Monteagudo E, MarcoAlgarra J: Initial experience with the Sophono Alpha 1 osseointegrated implant. Acta Otorrinolaringol Esp 2014;65:361-364.

Faber HT, de Wolf MJF, Cremers CWRJ, Snik AFM, Hol MKS: Benefit of Baha in the elderly with single-sided deafness. Eur Arch Otorhinolaryngol 2013;270:1285-1291. 
1 Finbow J, Bance M, Aiken S, Gulliver M, Verge J, Caissie R: A Comparison Between Wireless CROS and Bone-anchored Hearing Devices for Single-sided Deafness: A Pilot Study. Otol Neurotol 2015;36:819-825.

Hirsch A, Anderson H: Audiologic test results in 96 patients with tumours affecting the eighth nerve. A clinical study with emphasis on the early audiological diagnosis. Acta Otolaryngol Suppl 1980;369:1-26.

Hol MKS, Bosman AJ, Snik AFM, Mylanus EAM, Cremers CWRJ: Bone-anchored hearing aids in unilateral inner ear deafness: an evaluation of audiometric and patient outcome measurements. Otol Neurotol 2005;26:999-1006.

Hol MKS, Nelissen RC, Agterberg MJH, Cremers CWRJ, Snik AFM: Comparison between a new implantable transcutaneous bone conductor and percutaneous bone-conduction hearing implant. Otol Neurotol 2013;34:1071-1075.

Kitterick PT, O’Donoghue GM, Edmondson-Jones M, Marshall A, Jeffs E, Craddock L, et al.: Comparison of the benefits of cochlear implantation versus contra-lateral routing of signal hearing aids in adult patients with single-sided deafness: study protocol for a prospective within-subject longitudinal trial. BMC Ear Nose Throat Disord 2014;14:7.

Kurz A, Flynn M, Caversaccio M, Kompis M: Speech understanding with a new implant technology: a comparative study with a new nonskin penetrating Baha system. Biomed Res Int 2014;2014:416205.

Leterme G, Bernardeschi D, Bensemman A, Coudert C, Portal J-J, Ferrary E, et al.: Contralateral Routing of Signal Hearing Aid versus Transcutaneous Bone Conduction in Single-Sided Deafness. Audiol Neurootol 2015:22;20:251-260. 
1 Magliulo G, Turchetta R, Iannella G, di Masino RV, de Vincentiis M: Sophono Alpha System and subtotal petrosectomy with external auditory canal blind sac closure. Eur Arch Otorhinolaryngol 2014;8; DOI: 10.1007/s00405-014-3123-2

Martin TPC, Lowther R, Cooper H, Holder RL, Irving RM, Reid AP, et al.: The boneanchored hearing aid in the rehabilitation of single-sided deafness: experience with 58 patients. Clin Otolaryngol 2010;35:284-290.

Robinson K, Gatehouse S, Browning GG: Measuring patient benefit from otorhinolaryngological surgery and therapy. Ann Otol Rhinol Laryngol 1996;105:415422.

Saroul N, Nicolas S, Akkari M, Mohamed A, Pavier Y, Yoann P, et al.: Long-term benefit and sound localization in patients with single-sided deafness rehabilitated with an osseointegrated bone-conduction device. Otol Neurotol 2013;34:111-114.

Siegert R: Partially implantable bone conduction hearing aids without a percutaneous abutment (Otomag): technique and preliminary clinical results. Adv Otorhinolaryngol 2011;71:41-46.

Siegert R, Kanderske J: A new semi-implantable transcutaneous bone conduction device: clinical, surgical, and audiologic outcomes in patients with congenital ear canal atresia. Otol Neurotol 2013;34:927-934.

Snik A, Agterberg M, Bosman A: How to quantify binaural hearing in patients with unilateral hearing using hearing implants. Audiol Neurootol 2015;20 Suppl 1:44-47.

Stewart CM, Clark JH, Niparko JK: Bone-anchored devices in single-sided deafness. Adv Otorhinolaryngol 2011;71:92-102. 
1 Sylvester DC, Gardner R, Reilly PG, Rankin K, Raine CH: Audiologic and surgical outcomes of a novel, nonpercutaneous, bone conducting hearing implant. Otol Neurotol 2013;34:922-926.

Tringali S, Grayeli AB, Bouccara D, Sterkers O, Chardon S, Martin C, et al.: A survey of satisfaction and use among patients fitted with a BAHA. Eur Arch Otorhinolaryngol 2008;265:1461-1464.

Van Wanrooij MM, Van Opstal AJ: Contribution of head shadow and pinna cues to chronic monaural sound localization. J Neurosci 2004;28;24:4163-4171.

Van Zon A, Peters JPM, Stegeman I, Smit AL, Grolman W: Cochlear implantation for patients with single-sided deafness or asymmetrical hearing loss: a systematic review of the evidence. Otol Neurotol 2015;36:209-219.

Vermeire K, Van de Heyning P: Binaural hearing after cochlear implantation in subjects with unilateral sensorineural deafness and tinnitus. Audiol Neurootol 2009;14:163-171.

Verstraeten N, Zarowski AJ, Somers T, Riff D, Offeciers EF: Comparison of the audiologic results obtained with the bone-anchored hearing aid attached to the headband, the testband, and to the "snap" abutment. Otol Neurotol 2009;30:70-75.

Wazen JJ, Young DL, Farrugia MC, Chandrasekhar SS, Ghossaini SN, Borik J, et al.: Successes and complications of the Baha system. Otol Neurotol 2008;29:1115-1119.

Wazen JJ, Spitzer JB, Ghossaini SN, Fayad JN, Niparko JK, Cox K, et al.: Transcranial contralateral cochlear stimulation in unilateral deafness. Otolaryngol Head Neck Surg 2003;129:248-254. 
1 Zeitler DM, Snapp HA, Telischi FF, Angeli SI: Bone-anchored implantation for single-sided

2 deafness in patients with less than profound hearing loss. Otolaryngol Head Neck Surg

3 2012;147:105-111.

4

5

6

7

8 
$1 \quad$ Fig. 1. A: Comparison between Alpha $1^{\circledR}$ (on the left) and Alpha $2^{\circledR}$ (on the right) external

2 processor . and after placement in the retroauricular region of the Alpha $2^{\circledR}$ (B). Surgical view

3 of internal magnet in place in a right ear (C)
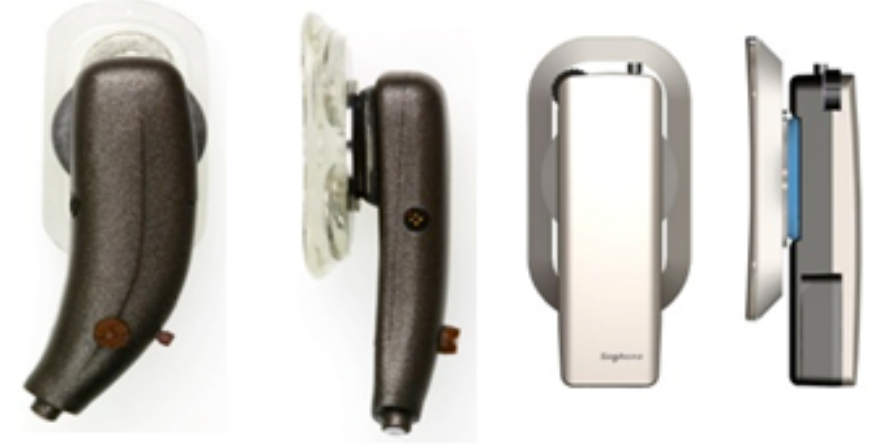

4 A

5
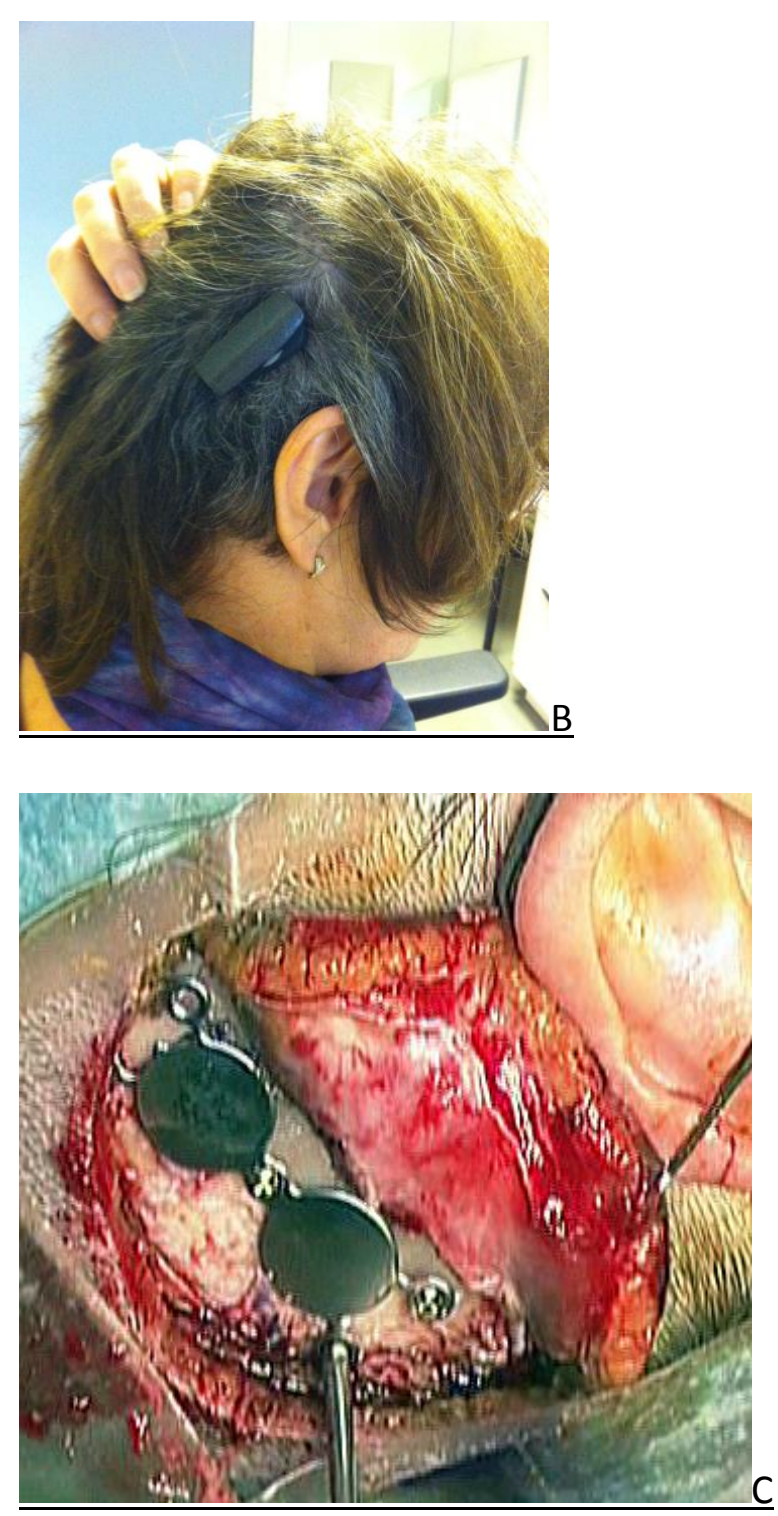
1 Fig. 2. (A) Free-field speech audiometry in quiet using monosyllabic words to the deaf side at

$240 \mathrm{~dB}$ and $60 \mathrm{~dB}$. The improvement of the speech discrimination score is significant with the

3 Alpha $2^{\circledR}$ compared to the Alpha $1^{\circledR}$ at $40 \mathrm{~dB}(\mathrm{p}=0.023$; paired t-test) white bars are test

4 performed in unaided condition, grey bars with Alpha $1^{\circledR}$ and black bars with Alpha $2^{\circledR}$

5 external processor). (B) Speech discrimination in noise; Results of the Hirsch (left column)

6 and squelch tests (right column) in unaided (white bars), with Alpha $1^{\circledR}$ (gray bars) and with

7 Alpha $2^{\circledR}$ (black bars). For the Hirsch test, the improvement between unaided condition and

8 Alpha 2 is significant $(\mathrm{p}<0.02$, paired t-test). For the squelch test, the improvement in the

9 speech reception threshold ( $\mathrm{SRT})$ is significant ( $\mathrm{p}=0.016$; paired t-test).

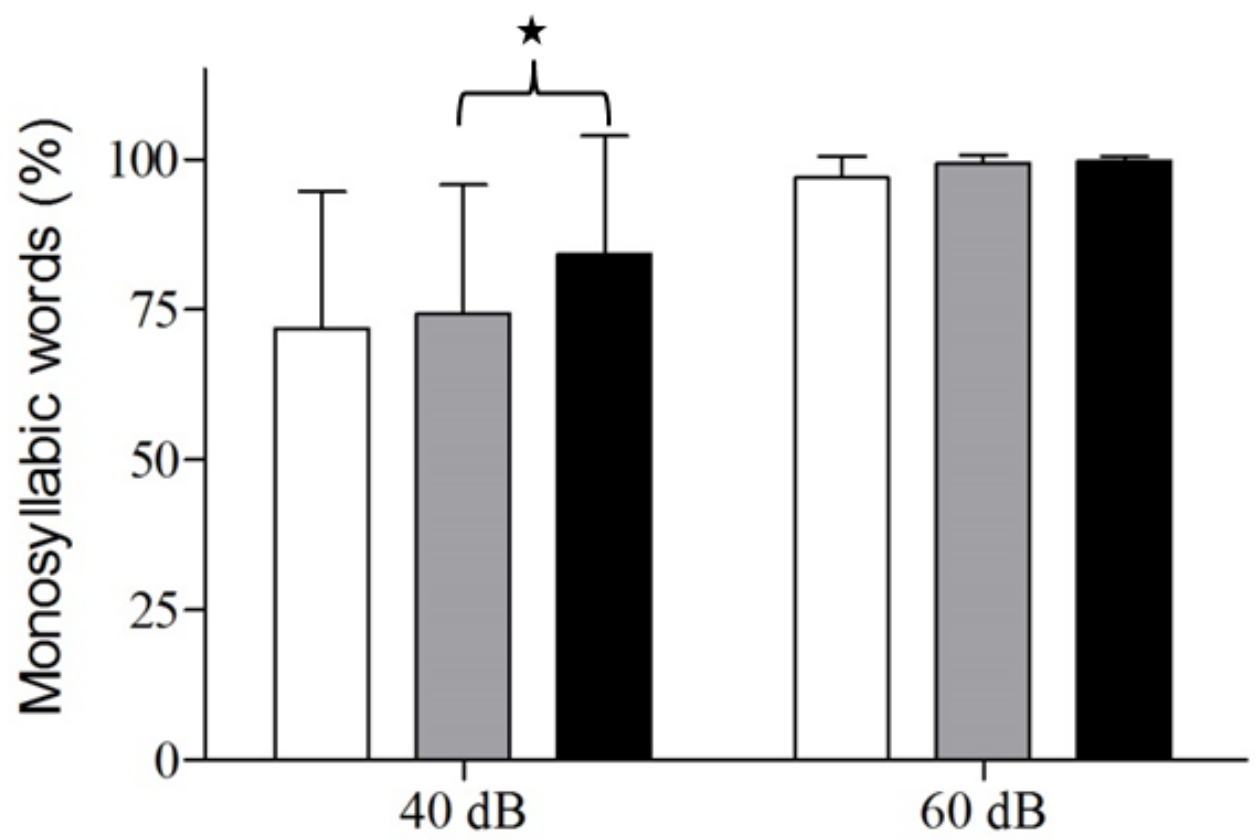




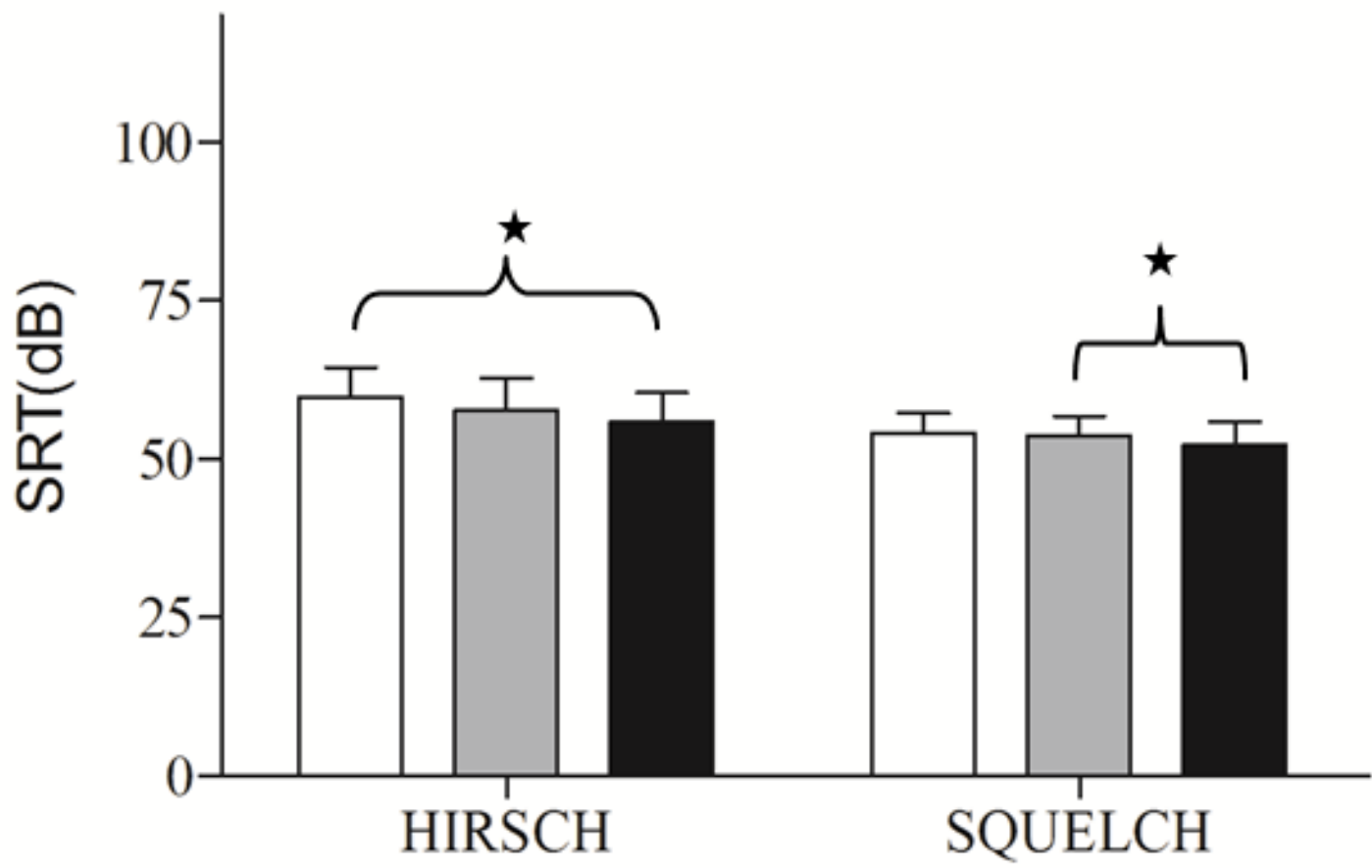

2 Fig. 4. Results of the Hirsch (left column) and squelch tests (right column) in unaided (white 3 bars), with Alpha $1^{\circledR}$ (gray bars) and with Alpha $2^{\circledR}$ (black bars). For the squelch test, the 4 difference between the 2 test is significant ( $\mathrm{p}=0.016$; SRT: speech reception threshold)

5

6

7

8

9

10 
1 Fig. 3. Glasgow Benefit Inventory (GBI) questionnaire showed no statistically significant

2 differences between the 2 devices (Wilcoxon non parametric test). Grey bars: with Alpha $1^{\circledR}$;

3 black bars: with Alpha $2^{\circledR}$.

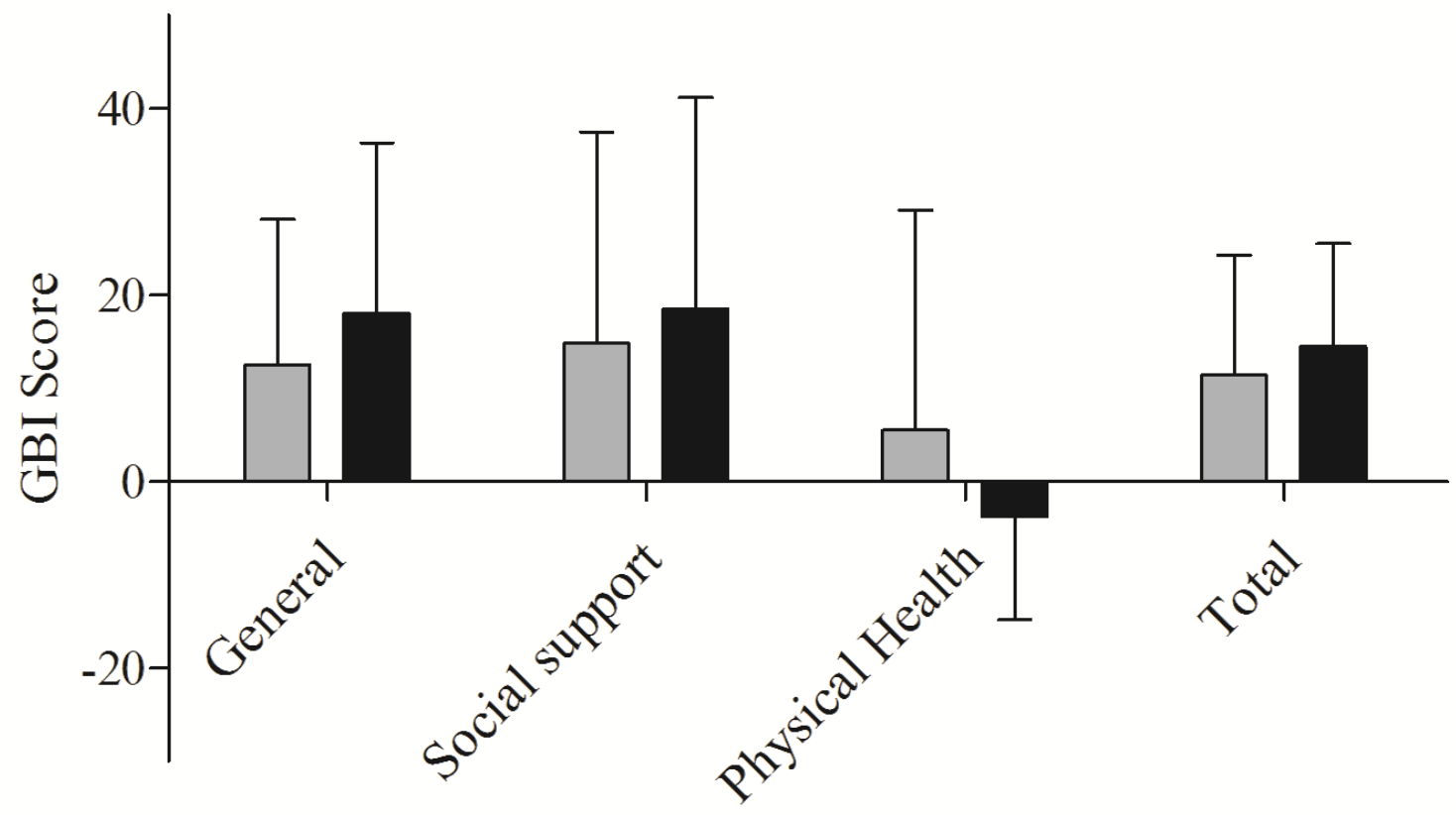

